\title{
Effect of age on Arden grating acuity
}

\author{
HAROLD W. SKALKA \\ From the Combined Programme in Ophthalmology, University of Alabama in Birmingham, \\ Eye Foundation Hospital, Birmingham, Alabama, USA
}

SUMMARY Arden grating test scores were obtained for 100 normal subjects under standardised testing conditions. While previously published ranges of interocular congruity were corroborated, a significant increase in test scores with increasing age was noted. This effect of age has not been previously reported. Physiological considerations suggest that such age dependency might have been expected. It is suggested that ranges of normal values be established for each of several age groups.

Arden (1978) has developed a practical clinical test of contrast sensitivity, commercially available as Arden Gratings. This book of 6 test plates displays vertical bar gratings of different frequencies (cycles/ degree), increasing in sinusoidal contrast as one moves down each plate. The variations in grating frequencies and contrasts allow testing of several points along the modulation transfer function (the graph of contrast sensitivity at different spatial frequencies). Contrast sensitivity has been shown to be a sensitive indicator of visual disturbance in many ophthalmological and neurological diseases (Bodis-Wollner, 1972; Sjøstrand and Frizen, 1977; Wolkstein et al., 1978), and the Arden Grating test has already demonstrated its clinical usefulness in early glaucoma (Arden and Jacobson, 1978) and minimal demyelinating disease (Arden and Gucukoglu, 1978).

This is a subjective test, and the numerical score achieved may be affected by several factors, such as ambient lighting, the rate of exposure of the grating pattern to the subject, the willingness of the subject to 'take a chance' (guess) or wait until certain before responding, and so on.

Despite the presence of such potential variables early clinical trials in an unsophisticated population (Minassian et al., 1978) show this test to be of great practical value when screening for ocular disease. Arden has reported that in the age range 11 to 70 there was no influence of age on the results, grouped in decades of age' (Arden, 1978). To the contrary, we have found that test scores rise (become worse) with increasing age and consider that age-corrected ranges of normal must be established for each set of examination protocols.

Correspondence to Harold W. Skalka, MD, 1720 Eighth Avenue South, Birmingham, Alabama 35233, USA.

\section{Patients and methods}

Hospital personnel and visitors free of ocular disease other than corrected refractive error and/or presbyopia were tested by the same examiner in the same room under identical lighting conditions. Visual acuities (with correction where appropriate) at the test distance (the patient's reading distance) were $\mathrm{J} 1$ or better. The plates were exposed at the same constant rate for all subjects. Tests were repeated only if anomalous results were obtained and, if not reproducible, were eliminated from tabulation. A total of 100 patients (200 eyes) were examined. Ages by decades were as follows: 8 patients were in their second decade, 38 in their third decade, 24 in their fourth, 14 in their fifth, and 16 were in their sixth decade; 87 patients were female, and 81 were Caucasian.

\section{Results}

Arden (1978) has stated that grating test scores greater than 82 indicate an abnormality. While most of our patients with higher scores were over $\mathbf{4 0}$ years of age, 6 under 40 had scores in one or both eyes exceeding 82. This may represent a difference in testing conditions. Indeed, our scores in general tended to be slightly higher than the averages obtained by Arden. Arden also states that interocular differences greater than 11 indicate an abnormality, and in this (a comparison of 2 eyes under identical test conditions) we basically agree. Only 7 of our patients showed interocular differences of greater than 11 (only 1 greater than 13, and on further testing this patient was found to have a microtropia in the eye that scored 14 points worse) (Fig. 1). However, we found a definite increase in test scores (decrease in contrast sensitivity) with increasing age 


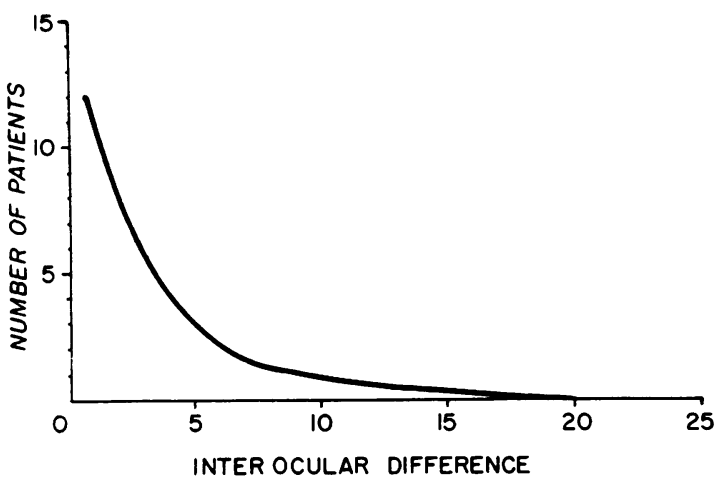

Fig. 1 Averaged curve of interocular difference in scores of subjects studied

(Fig. 2). Average interocular differences did not increase with advancing age. Average scores in the second decade were 69.16, with an average interocular difference (AID) of 4.06, 70.76 (AID 2.93) in the third decade, 73.20 (AID 4.64) in the fourth decade, 79.46 (AID 4.64) in the fifth decade, and 88.23 (AID 4.63) in the sixth decade.

\section{Discussion}

Although surprisingly little work has been done on visual changes with increasing age, it is known that visual acuity deteriorates after the age of about 45 years (Weale, 1975). This is not surprising in view of factors such as senile miosis, lenticular and other medial changes, cellular loss in the brain (and retina?) with ageing, etc. Physiological functions such as macular recovery after photostress have been shown to deteriorate with age (Severin $e t$ al., 1967; Henkind and Siegel, 1967). It would be surprising if contrast sensitivity remained unaffected. Indeed, results in our patients show contrast sensitivity thresholds to rise with age despite excellent Snellen acuity. Snellen optotypes are highcontrast targets. One would expect abnormalities in distinguishing such blatant targets to occur with more advanced stages of degenerative changes than one would hope to uncover with subtle variations in contrast.

We found advancing age to be associated with increase of scores in all test plates irrespective of spatial frequency, a result implicating the contrast function rather than acuity. Both coarse and fine gratings were seen by our older patients (on average) only when contrast was increased over that necessary for younger patients.

The Arden grating test is an elegantly simple method of evaluating an important and hitherto usually neglected parameter of visual function. However, as it is a subjective test requiring active participation by the patient, people administering the test should probably establish their own ranges of normal values until lighting and rate of presentation are standardised. Previously published values concerning interocular variability are probably generally valid. Lighting and rate of stimulus presentation should be essentially the same for both eyes, and internal test consistency should yield reproducibility in this regard. Normal values, however, definitely appear to be age dependent, at least in our patients. Advancing age affects nearly all areas of physiological performance, and

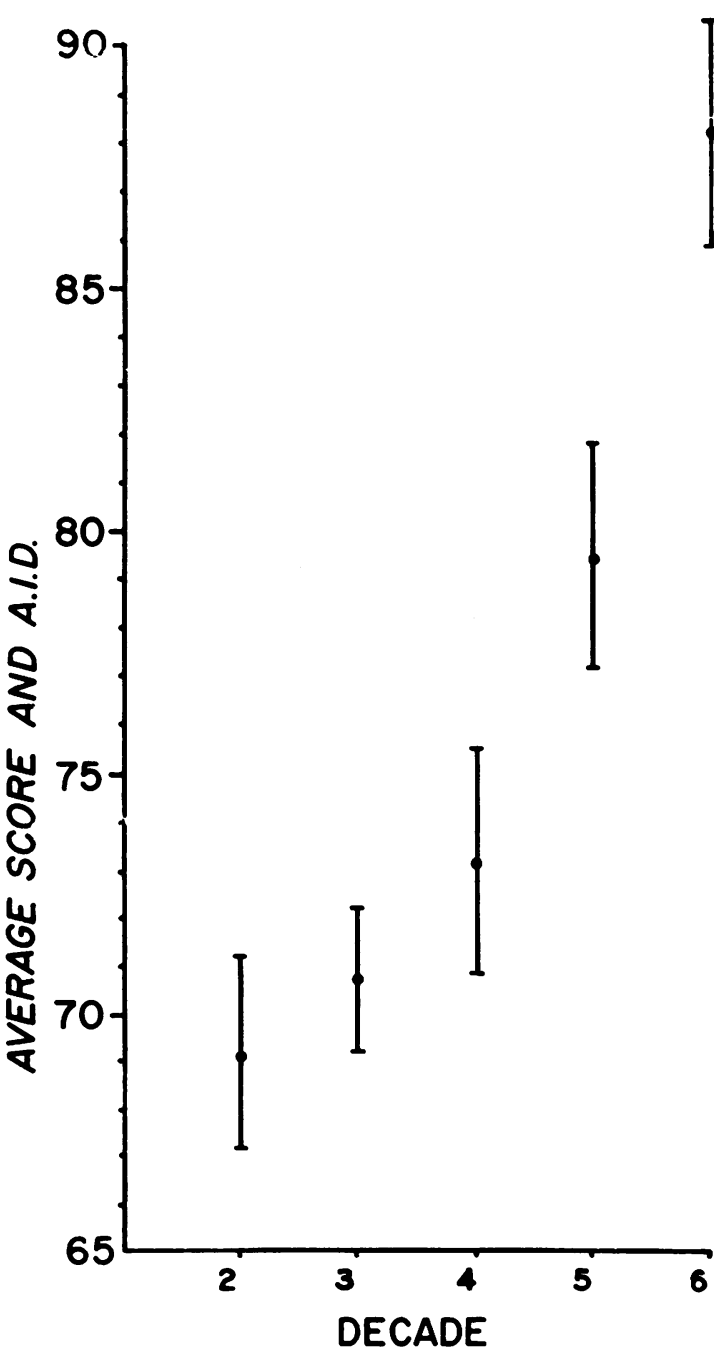

Fig. 2 Average score and average interocular difference (AID) by decade 
contrast sensitivity, as measured by the Arden Grating test, is no exception. Consequently, normal values must be developed for each age group.

I thank Judy Bacon, RN, for her skilled administrative and technical help in this study.

\section{References}

Arden, G. B. (1978). The importance of measuring contrast sensitivity in cases of visual disturbance. British Journal of Ophthalmology, 62, 198-209.

Arden, G. B., and Gucukoglu, A. G. (1978). Grating test of contrast sensitivity in patients with retrobulbar neuritis. Archives of Ophthalmology, 96, 1626-1629.

Arden, G. B., and Jacobson, J. (1978). A simple grating test for contrast sensitivity: preliminary results indicate value for screening in glaucoma. Investigative Ophthalmology, 17, 23-32.

Bodis-Wollner, I. (1972). Visual acuity and contrast sensi- tivity in patients with cerebral lesions. Science, 178, 769771.

Henkind, P., and Siegel, I. M. (1967). The scotometer. A device for measuring macular recovery time. American Journal of Ophthalmology, 64, 314-315.

Minassian, D. C., Jones, B. R., and Zargarizadeh, A. (1978). The Arden Grating test of visual function: a preliminary study of its practicability and application in a rural community in north-west Iran. British Journal of Ophthalmology, 62, 210-212.

Severin, S. L., Tour, R. L., and Kershaw, R. H. (1967). Macular function and the Photostress test I. Archives of Ophthalmology, 77, 2-7.

Sjøstrand, J., and Frizen, L. (1977). Contrast sensitivity in macular disease. Acta Ophthalmologica, 55, 507-514.

Weale, R. A. (1975). Senile changes in visual acuity. Transactions of the Ophthalmological Societies of the United Kingdom, 95, 36-38.

Wolkstein, M., Atkin, A., and Bodis-Wollner, I. (1978). Grating acuity in two sisters with tapetoretinal degeneration. Documenta Ophthalmologica (in press). 